\title{
Monitoring of Hemodynamics in Human Skin Using Pulsed Photothermal Radiometry and Optical Spectroscopy
}

\author{
by N. Verdel* and B. Majaron*,**
}

\author{
* Jožef Stefan Institute, Department for Complex Matter, Jamova 39, SI-1000 Ljubljana, Slovenia, \\ nina.verdel@ijs.si \\ ** Faculty of Mathematics and Physics, University of Ljubljana, Jadranska 21, SI-1000 Ljubljana, Slovenia \\ boris.majaron@ijs.si
}

\begin{abstract}
We present a test of our novel methodology for noninvasive quantitative characterization of human skin in vivo involving a customary pressure-cuff test in healthy volunteers at different cuff pressures. Our experimental approach combines time-resolved measurements of mid-infrared emission from sample surface after exposure to a short light pulse and diffuse reflectance spectroscopy in visible part of the spectrum. Both data sets are fitted simultaneously with predictions of a dedicated numerical model of light transport in a four-layer skin model (i.e., inverse Monte Carlo). This approach allows assessment of, e.g., blood contents and oxygenation levels in papillary and reticular dermis.
\end{abstract}

\section{Introduction}

We have recently introduced a novel methodology for quantitative assessment of structure and composition of human skin in vivo. The approach combines pulsed photothermal radiometry (PPTR), which involves time-resolved measurements of mid-infrared emission from sample surface after exposure to a short light pulse, and diffuse reflectance spectroscopy (DRS) in visible part of the spectrum.

The experimental data are fitted with predictions of a dedicated numerical model of light transport in strongly scattering medium (Monte Carlo) using a four-layer model of skin. This approach allows assessment of the epidermal and dermal thickness, chromophore contents (e.g., melanin, oxy- and deoxy-hemoglobin), as well as scattering properties of both skin and subcutis [1-3].

We have recently performed an objective, albeit only partial test of the described approach, based on a comparison of the assessed epidermal thicknesses with the evidence obtained using multi-photon microscopy, which provides optical sectioning capability [2]. In the following, we report on a complementary test of the same technique, in which we compare the results from intact skin in a healthy volunteer with those obtained after application of a bloodpressure cuff at different pressures at the same test site.

\section{Study protocol}

\subsection{Experimental setup}

The presented example involves one healthy volunteer with fair complexion (female, age 28). Her blood pressure was established to be $110 / 70 \mathrm{~mm} \mathrm{Hg}$ (systolic/diastolic). According to these values, our measurements were performed on the dorsal side of the volunteer's forearm upon application of a blood-pressure cuff inflated to 80,100, and $120 \mathrm{~mm} \mathrm{Hg}$ for a period of 3 minutes. This should result in a gradual transition from partial (venous) to total occlusion of the peripheral blood flow. The same measurements were performed at the same test site before administration of the pressure cuff, in order to obtain baseline values characteristic for intact skin.

Prior to PPTR measurements, the test site was prepared by removing the dehydrated superficial layer of skin (stratum corneum) by tape stripping, to ensure unobstructed heat transport to the skin surface. The site was then irradiated with a single $1 \mathrm{~ms}$ laser pulse at $\lambda=532 \mathrm{~nm}$ from a medical-grade laser (DualisVP by Fotona d.o.o., Slovenia). The applied light is absorbed well by both epidermal melanin and hemoglobin in dermal vasculature. The radiant exposure was estimated to $\sim 0.30 \mathrm{~J} / \mathrm{cm}$ at the effective spot size of $\sim 5 \mathrm{~mm}[1-3]$.

Mid-IR emission from the tissue surface was recorded with a fast IR camera (SC7500 by FLIR Systems Inc.; $\lambda=$ 3.5-5.1 $\mu \mathrm{m}$ ) at a rate of 1000 frames per second. PPTR signals were obtained by lateral averaging of the radiometric data over a suitable area of interest (typically $1 \times 1 \mathrm{~mm}^{2}$ ) and subtracting the baseline value [1-3]. The manufacturer provided calibration system (Hypercal ${ }^{\mathrm{TM}}$ ) was used for conversion of raw signal amplitudes to radiometric temperature values.

The DRS in visible spectral range $(\lambda=400-700 \mathrm{~nm})$ were measured using an integrating sphere (IS) with an internal light source and sample opening diameter of $10.3 \mathrm{~mm}$ (ISP-REF by Ocean Optics). Spectral response of the spectrometer (USB4000, Ocean Optics) was calibrated using a Spectralon ${ }^{\odot}$ white standard (Labsphere, North Sutton, NH). Prior to data analysis, the so-called single-beam substitution error was removed from the raw DRS by applying an algebraic correction, developed earlier based on both theoretical analysis and experimental verification [4]. 


\subsection{Data analysis}

The structure and composition of skin at the test site are assessed by fitting simultaneously the PPTR signals and DRS spectra with the corresponding predictions from a dedicated numerical model of light transport and energy deposition in human skin, using the established weighted-photon Monte Carlo technique [5]. Only the initial $1.5 \mathrm{~s}$ of the transient PPTR signals are considered in the analysis, and the DRS values are analyzed at only 13 wavelengths (between 410 and $600 \mathrm{~nm}$, selected based on most prominent features in the absorption spectra) to ease the computational load. Nevertheless, the process is parallelized using the CUDA technology and run on a high-performance graphics card.

The applied optical model of skin assumes four optically homogeneous layers, representing the epidermis, papillary and reticular dermis, and subcutis (adipose; see figure 1). Determination of the absorption properties of each layer in relation to the fractional contents of the included chromophores was described in detail earlier $[1-3,6]$.

In addition to the thicknesses of the constitutive skin layers and fractional contents of the specific absorbers, we also allow individual adjustment of dermal scattering properties according to the customary ansatz [1,2]:

$$
\mu_{\mathrm{s}}^{\prime}(\lambda)=a\left(\frac{\lambda}{500 \mathrm{~nm}}\right)^{-p}
$$

Moreover, because literature data on scattering properties of the epidermis are rather sparse and inconsistent, we apply the same ansatz also for the epidermis, but with two times higher amplitude (i.e., 2 a) $[1,2]$. The amplitude of scattering in subcutaneous adipose tissue is also fitted individually, albeit using a different spectral ansatz [7], while its absorption spectrum is fixed, based on literature data [8]. The refractive indices are set to 1.45 for the epidermis, 1.37 for dermis, and 1.34 for subcutis.

With the described four-layer model of skin, the best match with experimental data is thus sought by varying the epidermal and dermal thickness $\left(d_{\mathrm{epi}}, d_{\mathrm{der}}\right)$, epidermal melanin content $(m)$, fractional blood contents in epidermis, papillary, and reticular dermis $\left(b_{\text {epi }}, b_{\text {pap }}\right.$ and $b_{\text {der }}$ ), papillary and reticular blood oxygenation levels ( $S_{\text {pap }}$ and $\left.S_{\text {ret }}\right)$, dermal scattering amplitude $(a)$ and power $(p)$, and subcutis scattering amplitude $(A)$. This is performed by objective multi-dimensional optimization using a nonlinear least-squares algorithm, implemented as a function Isqnonlin in MATLAB (Mathworks Inc., Natick, MA). The thickness of the papillary dermis is fixed at $0.100 \mathrm{~mm}$ to help improve the robustness of the inverse analysis.

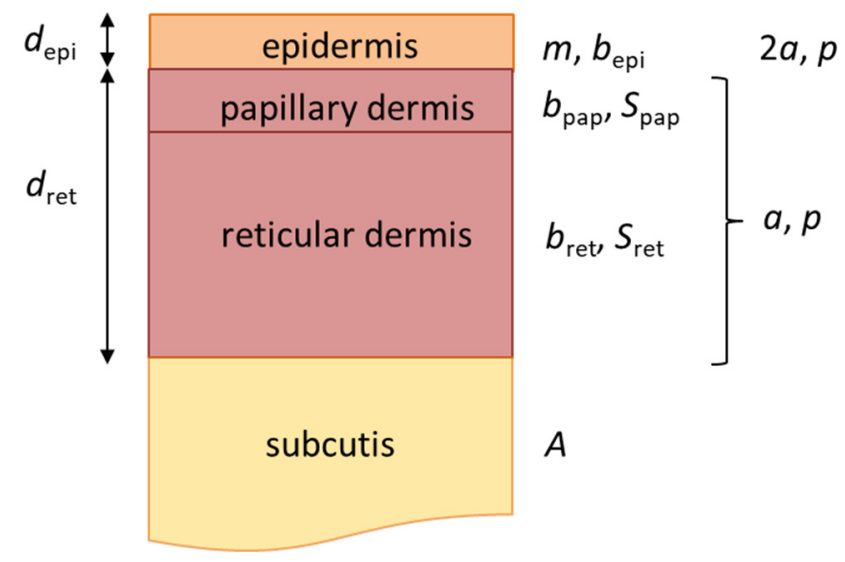

Fig. 1. Schematic representation of our four-layer skin model with 11 free parameters (see the text for explanations).

\section{Results}

Figure 2 presents the PPTR signals and DRS as measured before (orange lines) and during occlusion of the peripheral blood flow imposed by a pressure cuff inflated to 80,100 , and $120 \mathrm{~mm} \mathrm{Hg}$ (see the legend).

All results obtained upon occlusion of the blood flow (i.e., cuff pressures of 80,100 , and $120 \mathrm{~mm} \mathrm{Hg}$ ) feature a significant increase of the PPTR signal amplitude (fig. 2a) and reduced diffuse reflectance across the included spectral range (fig. 2b). This is consistent with the fact that the blood content in skin is increased during the pressure-cuff test, which leads to enhanced absorption of incident light. In addition, the double absorption peak characteristic for oxyhemoglobin which is prominent in intact skin (at 541 and $577 \mathrm{~nm}$; orange line in fig. 2b) is gradually transforming into a single absorption band (at $555 \mathrm{~nm}$; indicative of deoxyhemoglobin) with the cuff pressure increasing from 80 to $120 \mathrm{~mm}$ $\mathrm{Hg}$ (blue). 

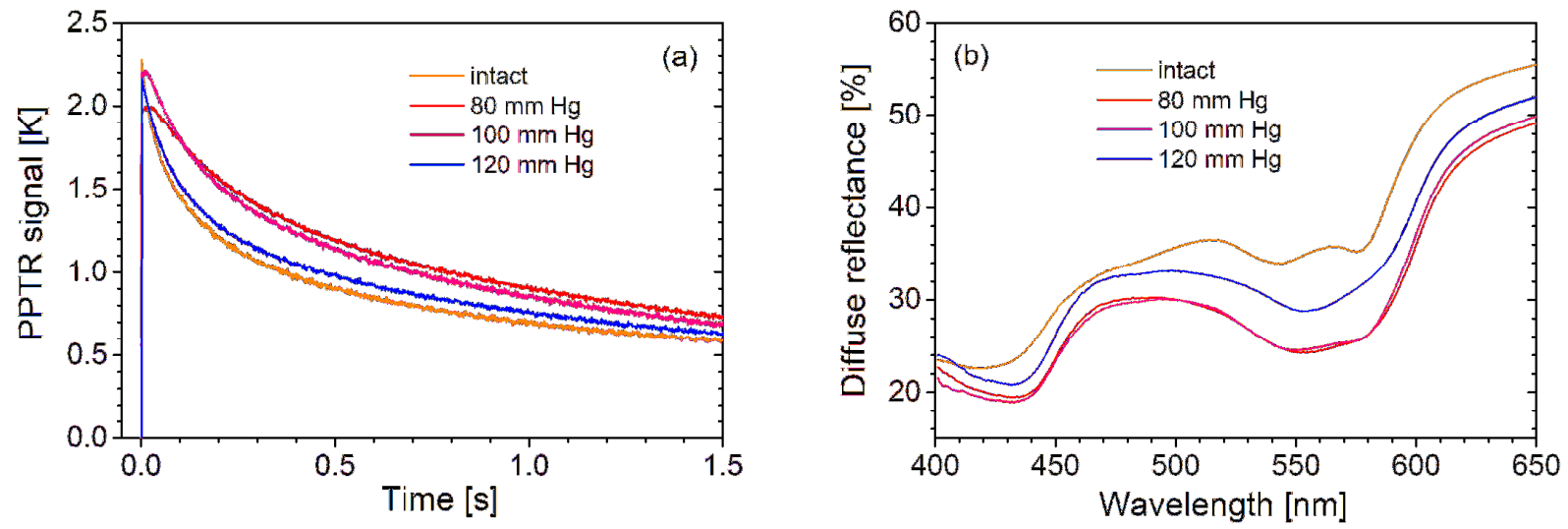

Fig. 2. PPTR signals (a) and DRS spectra (b) as measured in intact human skin (orange lines) and upon occlusion of the peripheral blood flow imposed by a pressure cuff inflated to 80 (red), 100 (pink), and $120 \mathrm{~mm} \mathrm{Hg} \mathrm{(blue).}$

In figure 3, we compare the PPTR signals and DRS spectra as obtained before (orange lines) and during the pressure-cuff test at $80 \mathrm{~mm} \mathrm{Hg}$ (red) with the corresponding best-fitting model predictions (dashed lines, blue). The match is very satisfactory, especially for the PPTR signals (figure 2a). Note that the small overshoot in the model DRS spectra at 470-510 nm can most likely be attributed to presence of chromophores such as beta-carotene and bilirubin, which are not included in our optical model.
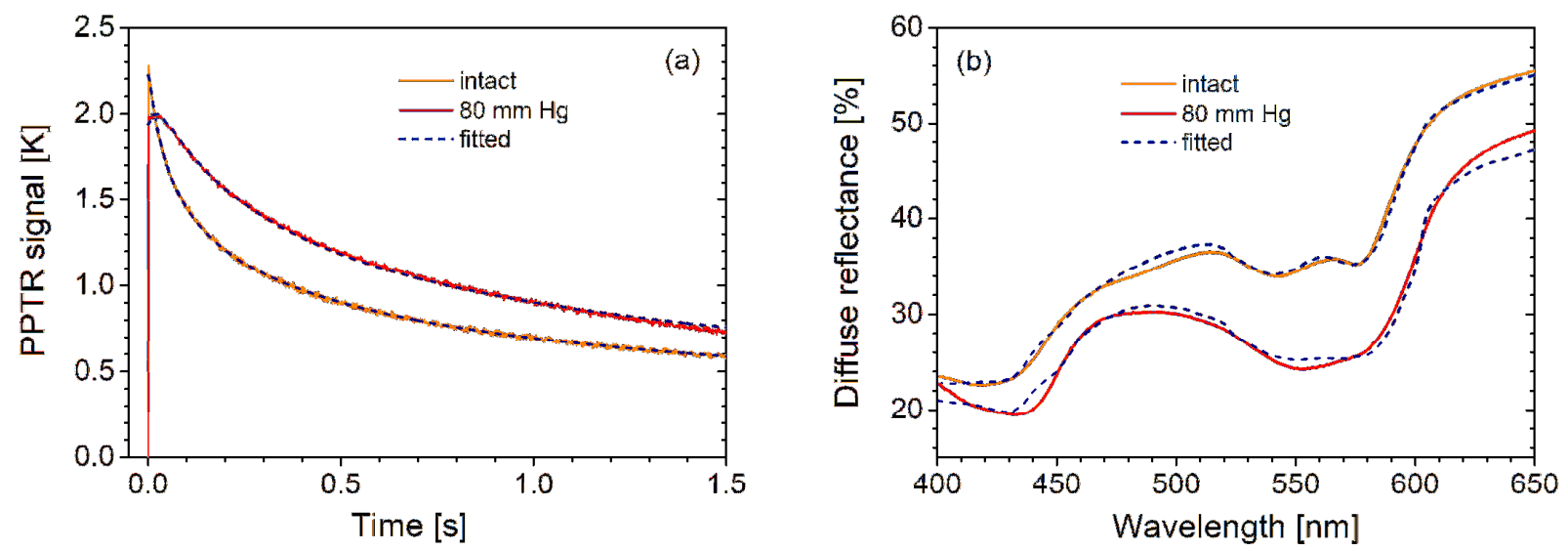

Fig. 3. PPTR signals (a) and DRS spectra (b) as measured in intact human skin (orange lines) and upon partial (venous) occlusion of the blood flow induced by the pressure cuff inflated to $80 \mathrm{~mm} \mathrm{Hg}$ (red). The best fitting model predictions are presented by dashed lines (blue).

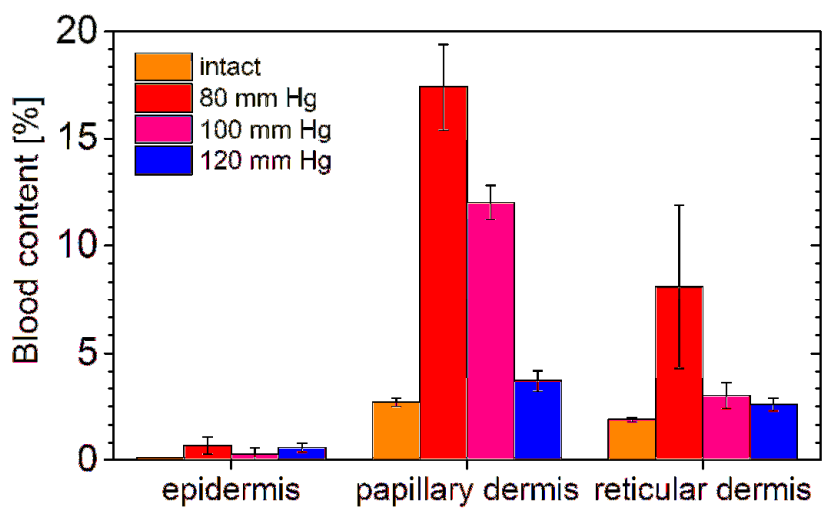

Fig. 4. Changes of blood content in epidermis (left), papillary (middle) and reticular (right) dermis within the intact skin (orange bars) and upon the occlusion by the pressure cuff at 80, 100, and $120 \mathrm{~mm} \mathrm{Hg}$ (see the legend). 


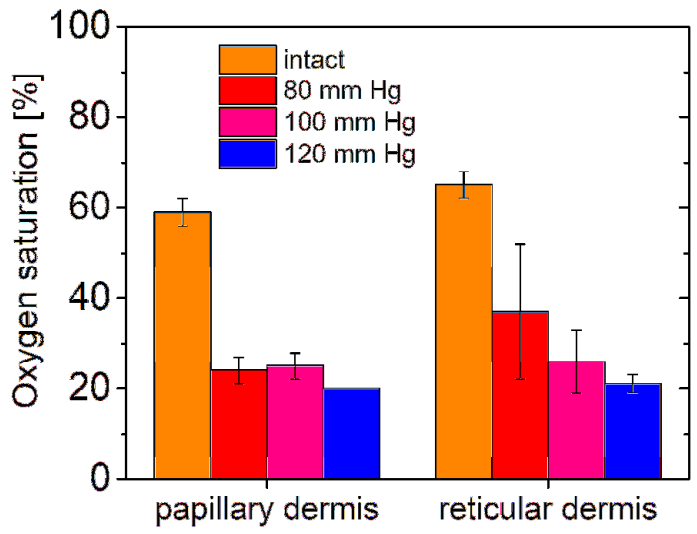

Fig. 5. Changes of oxygen saturation in papillary (left) and reticular dermis (right) within the intact skin (orange bars) and upon the occlusion by the pressure cuff at 80, 100, and $120 \mathrm{~mm} \mathrm{Hg}$ (see the legend).

Table 1. Skin model parameter values as assessed from experimental data.

\begin{tabular}{|c|c|c|c|c|}
\hline Skin model parameter & Intact skin & $80 \mathrm{~mm} \mathrm{Hg}$ & $100 \mathrm{~mm} \mathrm{Hg}$ & $120 \mathrm{~mm} \mathrm{Hg}$ \\
\hline epidermal thickness [mm] & $0.09 \pm 0.01$ & $0.11 \pm 0.03$ & $0.09 \pm 0.01$ & \pm 0.01 \\
\hline melanin content [vol. \%] & 1.4 & 1.1 & 1.5 & 1.3 \\
\hline epidermal blood content [vol. \%] & 0.1 & $0.7 \pm 0.4$ & \pm 0.2 & \pm 0.2 \\
\hline dermal blood content - papillary [vol. \%] & $2.7 \pm 0.2$ & \pm 2.0 & \pm 0.8 & \pm 0.5 \\
\hline dermal blood content - reticular [vol. \%] & \pm 0.1 & \pm 5.8 & \pm 0.6 & \pm 0.3 \\
\hline blood oxygenation - papillary dermis [\%] & \pm 3 & \pm 4 & \pm 3 & 20 \\
\hline blood oxygenation - reticular dermis [\%] & \pm 3 & \pm 15 & \pm 7 & 21 \\
\hline dermal thickness $[\mathrm{mm}]$ & 0.53 & 0.52 & 0.51 & $0.55 \pm 0.10$ \\
\hline dermal scattering amplitude (a) $\left[\mathrm{mm}^{-1}\right]$ & \pm 0.3 & \pm 0.8 & \pm 0.3 & \pm 0.1 \\
\hline dermal scattering power $(p)$ & \pm 0.2 & \pm 0.4 & \pm 0.2 & \pm 0.1 \\
\hline adipose scattering parameter & 1.1 & 0.3 & 0.7 & 0.9 \\
\hline
\end{tabular}

As can be seen from our results, compiled in table 1, the extracted skin parameter values corresponding to intact healthy skin are within physiologically plausible ranges. This supports our earlier experience with the same or very similar analyses, also including other volunteers $[1-3,9,10]$. Specifically, e.g., the fractional blood content is higher in the papillary as compared to reticular dermis, while the oxygenation level in the former is lower, in agreement with earlier reports from other groups [11,12].

Of most interest in this study, however, are the clearly indicated substantial rises of the dermal blood contents upon application of the pressure cuff (see figure 4). This effect is especially prominent at the cuff pressures of 80 and 100 $\mathrm{mm} \mathrm{Hg}$, which obstruct blood flow through the veins but not also through the arteries, thus leading to pronounced blood containment in the skin.

The above effect is evidenced also by the considerable decrease of the blood oxygenation level, which drops from $\sim 60 \%$ in intact skin to $\sim 25 \%$ at the cuff pressure of $100 \mathrm{~mm} \mathrm{Hg}$ (figure 5).

At the cuff pressure of $120 \mathrm{~mm} \mathrm{Hg}$, in contrast, both venous and arterial blood flow are expected to be completely obstructed, based on the volunteer's blood pressure readings of $110 / 70 \mathrm{~mm} \mathrm{Hg}$. Consequently, the increase in dermal blood contents relative to intact skin is smaller than that seen upon venous occlusion discussed just above. Meanwhile, the blood oxygenation levels are even lower in comparison, again in agreement with other experiments involving total blood occlusion $[1,10]$.

It is also important to note that the assessed values of the epidermal and dermal thickness assume nearly constant values throughout the experimental protocol (figure 6), despite the fact that the four data sets were analyzed independently. This demonstrates the robustness of our methodology, as these values are not expected to vary significantly upon partial or total blood occlusion. 

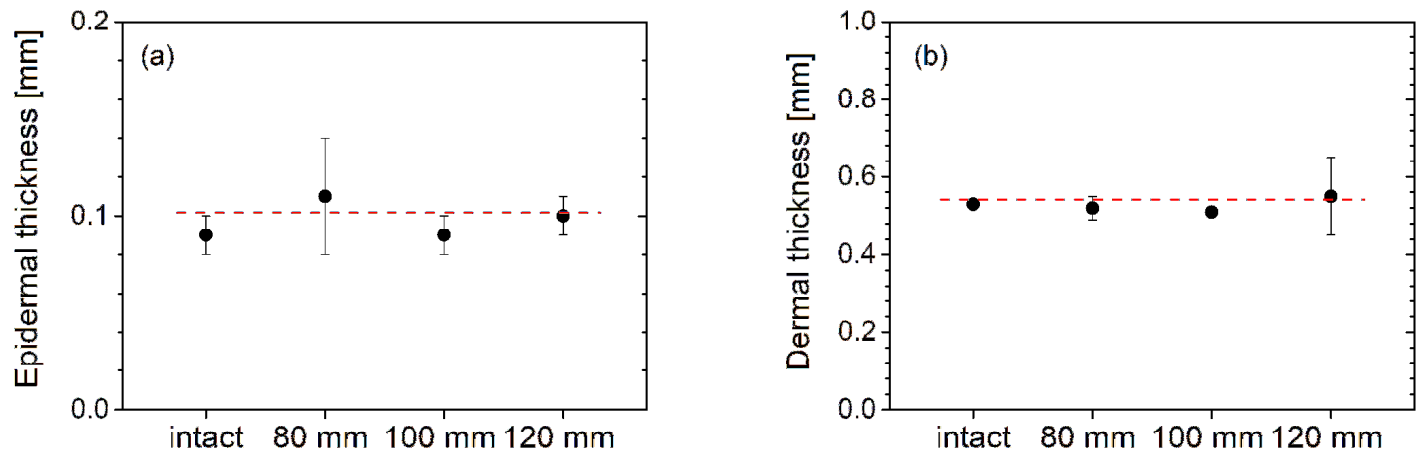

Fig. 6. Epidermal thickness (a) and dermal thickness (b) as obtained by fitting model predictions within the intact healthy skin and upon the occlusion by the pressure cuff at 80, 100, and $120 \mathrm{~mm} \mathrm{Hg}$.

Similarly, the melanin content, dermal scattering amplitude $(a)$ and scattering power $(b)$ remain nearly constant within the estimated error margins, although their fluctuations may be indicating that there is some room for improvement in terms of its robustness of the involved inverse analysis. Based on preliminary evidence, some improvement might be gained by simultaneous analysis of data from different time points (e.g., intact and obstructed blood flow in the discussed example) with selected parameter values bound to assume the same value $[1,7]$.

\section{REFERENCES}

[1] N. Verdel, A. Marin, L. Vidovič, et al., Analysis of hemodynamics in human skin using photothermal radiometry and diffuse reflectance spectroscopy. Proceedings of SPIE vol. 10412, paper 1041300; ECBO 2017, Muenchen (Germany) 2017.

[2] N. Verdel, G. Lentsch, M. Balu, B. J. Tromberg, B. Majaron, Noninvasive assessment of skin structure by combined photothermal radiometry and optical spectroscopy: coregistration with multiphoton microscopy. Applied Optics; 57 (18): pp. D117-D122, 2018.

[3] N. Verdel, A. Marin, L. Vidovič, et al., In vivo characterization of structural and optical properties of human skin by combined photothermal radiometry and diffuse reflectance spectroscopy. Proceedings of SPIE vol. 10037, paper $100370 \mathrm{H}$; BiOS 2017, San Francisco (USA) 2017.

[4] L. Vidovič, B. Majaron, Elimination of single-beam substitution error in diffuse reflectance measurements using an integrating sphere. Journal of Biomedical Optics; 19 (2): pp. 027006-1-9, 2014.

[5] L. Wang, S. L. Jacques, L. Zheng, MCML-Monte Carlo modeling of light transport in multi-layered tissues. Computational Methods and Programs in Biomedicine; 47 (2): 131-146, 1995.

[6] M. Milanič, B. Majaron, Three-dimensional Monte Carlo model of pulsed-laser treatment of cutaneous vascular lesions. Journal of Biomedical Optics; 16 (12): pp. 128002-1-12, 2011.

[7] P. Naglič, L. Vidovič, M. Milanič, et al., Applicability of diffusion approximation in analysis of diffuse reflectance spectra from healthy human skin. Proceeding of SPIE 9032, paper 90320N; Biophotonics Riga (Latvia) 2013.

[8] C. R. Simpson, M. Kohl, M. Essenpreis, et al., Near-infrared optical properties of ex vivo human skin and subcutaneous tissues measured using the Monte Carlo inversion technique. Physics in Medicine and Biology; 43 (9): pp. 2465-2478, 1998.

[9] L. Vidovič, M. Milanič, and B. Majaron, Objective characterization of bruise evolution using photothermal depth profiling and Monte Carlo modeling. Journal of Biomedical Optics; 20 (1): pp. 017001-1-11, 2015.

[10] A. Marin, N. Verdel, L. Vidovič, et al., Assessment of individual bruising dynamics by pulsed photothermal radiometry and inverse Monte Carlo analysis. Proceedings of SPIE vol. 10412, paper 104130P; ECBO 2017, Muenchen (Germany) 2017.

[11] L. F. Douven, G. W. Lucassen, Retrieval of optical properties of skin from measurement and modeling the diffuse reflectance. Proceedings of SPIE vol. 3914, pp. 312-323; BiOS 2000, San Jose, CA (USA) 2000.

[12] X. Chen, W. Lin, C. Wang, et al., In vivo real-time imaging of cutaneous hemoglobin concentration, oxygen saturation, scattering properties, melanin content, and epidermal thickness with visible spatially modulated light. Biomedical Optics Express; 8 (12): 5468-5482, 2017. 